\title{
Temporal and angular variation of the solar limb brightening at $17 \mathrm{GHz}$
}

\author{
C. L. Selhorst ${ }^{1}$, A. V. R. Silva ${ }^{2}$, J. E. R. Costa $^{1}$, and K. Shibasaki ${ }^{3}$ \\ 1 CRAAM, Instituto Nacional de Pesquisas Espaciais, São José dos Campos, SP 12201-970, Brasil \\ 2 CRAAM, Universidade Presbiteriana Mackenzie, São Paulo, SP 01302-907, Brasil \\ 3 Nobeyama Radio Observatory, Minamimaki, Minamisaku, Nagano 384-1305, Japan \\ Received 19 November 2002 / Accepted 19 December 2002
}

\begin{abstract}
In order to better understand the atmosphere structure of the Sun, we have analyzed over 3000 daily maps of the Sun taken at $17 \mathrm{GHz}$ from the Nobeyama Radioheliograph (NoRH) from 1992 through 2001, focusing on the excess brightness temperature observed near the limb. The purpose of this work is to characterize the limb brightness in two ways: (i) study the temporal variation of the intensity and radial width of polar brightening; and (ii) measure the brightness distribution along the limb as a function of position angle and compare it with data at other wavelengths throughout the solar cycle. The mean intensity of the polar regions were found to be approximately $13 \%$ and $14 \%$ above quiet Sun levels at the North and South poles, respectively. Moreover, the polar brightenings are strongly anti-correlated with solar activity (as measured by sunspot number). The radial width of the excess brightness is slightly over 1 arcmin for both polar regions. Only a small variation with the solar cycle was observed during the decline of last maximum, that is, the Southern polar brightening was found to be both wider and brighter than the Northern one for the 23rd cycle. As for the angular variation of the limb brightening, for a month during a period of minimum activity, it reaches $25 \%$ above quiet Sun levels at the poles, $\sim 15 \%$ near the equator, and $10 \%$ at intermediate regions. $\mathrm{H} \alpha$ images also show brightening enhancements at the polar regions for the same period. We also found a strong anti-correlation between the radio polar brightenings and the coronal holes seen in soft X-ray images from 1992 to 2001. There seems to be a strong association of the radio limb brightening at $17 \mathrm{GHz}$ with faculae. The implications of these correlations are discussed.
\end{abstract}

Key words. Sun: general - Sun: corona - Sun: chromosphere

\section{Introduction}

The study of the brightness temperature variation throughout the solar disc, obtained from radio observations (at microwave, millimetric, and submillimetric wavelengths), has fundamental implications in the determination of chromospheric and coronal temperature and density structures. Theoretical models predict a brightening near the solar limb at high radio frequencies $(\gtrsim 15 \mathrm{GHz})$ caused by the positive temperature gradient of the solar atmosphere. Table 1 lists a sample of positive detection of temperature excess brightening from $17 \mathrm{GHz}$ to submillimetric wavelengths. These results show a limb excess of temperature varying from 2 to $20 \%$ above quiet Sun levels within approximately 1 arcmin of the limb, with no clear dependence on wavelength.

Nevertheless, many authors have also reported the absence of such brightening, for example at $25 \mathrm{GHz}$ (Furst et al. 1979), $35 \mathrm{GHz}$ (Kawabata et al. 1980), $88 \mathrm{GHz}$ (Simon \& Zirin 1969; Joensen et al. 1974), $98 \mathrm{GHz}$ (Kosugi et al. 1986), $100 \mathrm{GHz}$ (Belkora et al. 1992), and $114 \mathrm{GHz}$ (Wannier et al. 1983). Usually, the presence of jets, called spicules, has been blamed

Send offprint requests to: C. L. Selhorst, e-mail: caius@craam.mackenzie.br for the lack of brightening detection (Simon \& Zirin 1969; Wannier et al. 1983).

Spicules are an important component of the solar chromosphere and probably affect the limb brightening observed at very high radio frequencies. At the limb, they are seen as jets of gas and are observed at chromospheric lines such as $\mathrm{H} \alpha$. The spicules have an uprising mass flux of approximately 100 times larger than the solar wind. The influence of the spicules on limb brightening has been widely discussed. As mentioned above, the lack of brightening predicted by theoretical models at frequencies $>15 \mathrm{GHz}$ has been attributed to the presence of spicules, which block part of the emission. On the other hand, at very high frequencies, Lindsey et al. (1981) argued that the gradual brightening observed at $860 \mathrm{GHz}$ was due to chromospheric material below the height of spicules, whereas Horne et al. (1981) proposed that at $235 \mathrm{GHz}$, the increase of the solar radius was due to optically thick spicules, which in turn caused the brightening.

Another atmospheric feature which might contribute to the emission at high radio frequencies are faculae. Faculae are small bright structures (sizes of a few arcsec) observed in the photosphere. Models have described faculae as thin magnetic flux tubes, with "hot walls" (Spruit 1976) or a "hot cloud" 
Table 1. Limb brightness excess temperature at high radio frequencies.

\begin{tabular}{cccl}
\hline \hline$v(\mathrm{GHz})$ & Brightening $\%$ & Width (arcmin) & Reference \\
\hline 17 & $10-15$ & & 1,2 \\
$33-37$ & $3-10$ & 2 & 3,4 \\
$87-89$ & $2-10$ & 0.5 & 5,6 \\
91 & 20 & & 8 \\
230 & $5-15$ & & 9,10 \\
250 & 2 & $1-2$ & $10,11,12,13$ \\
$350-366$ & $10-16$ & 1 & 10,14 \\
$857-860$ & $3-5$ & &
\end{tabular}

${ }^{1}$ Shibasaki (1998); ${ }^{2}$ Nindos et al. (1999); ${ }^{3}$ Kundu et al. (1977); ${ }^{4}$ Kosugi et al (1986); ${ }^{5}$ Gomez-Gonzalez et al. (1983); ${ }^{6}$ Pohjolainen (2000); ${ }^{7}$ Shimabukuro et al. (1975); ${ }^{8}$ Horne et al. (1981); ${ }^{9}$ Clark \& Park (1968); ${ }^{10}$ Lindsey et al. (1995); ${ }^{11}$ Lindsey et al. (1984);

${ }^{12}$ Bastian et al. (1993); ${ }^{13}$ Ewell et al. (1993); ${ }^{14}$ Lindsey et al. (1981).

(Chapman 1979). Faculae are mainly observed within active regions and near the poles. Several authors have reported a significant increase in the number of faculae toward the poles (Ortiz et al. 2002; Erofeev 2001), and the intensity contrast of faculae has been shown to increase near the poles (Wang \& Zirin 1987; Lawrence 1988; Unruh et al. 1999 and references therein).

Several authors have reported the existence of regions of enhanced temperatures at radio wavelengths in the polar zones of the Sun (Kundu \& McCullogh 1972; Efanov et al. 1980; Riehokainen et al. 1998, 2001; Shibasaki 1998; Nindos et al. 1999; Gelfreikh et al. 2002). Riehokainen et al. (1998) and Gelfreikh et al. (2002) showed that these bright radio features could be divided into two groups: low latitude $\left(<40^{\circ}\right)$ brightenings associated with active regions and high latitude $\left(>40^{\circ}\right)$ regions that are uncorrelated with the sunspot cycle.

So far, most limb studies at radio frequencies were done on sporadic data taken over a short period of time, of the order of a few days. Hence, no long term study has been performed on the dependence of the brightening with the solar cycle, with few exceptions. Efanov et al. (1980) concluded that the polar brightening was anti-correlated with the solar activity cycle.

Moreover, there is very little information on the angular variation of the limb brightening, since most previous work is based on North-South scans. Shibasaki (1998), from analysis of 5 years of NoRH data, concluded that the observed brightening at $17 \mathrm{GHz}$ was the sum of two components: one of the order of $10 \%$ due to the temperature gradient of the atmosphere and a second one up to $40 \%$ due to bright patches near the poles. Shibasaki (1998) did not verify a one-to-one correlation between the polar brightening at $17 \mathrm{GHz}$ and coronal holes observed in soft X-ray images. Bastian et al. (1993) measured a $16 \%$ limb brightening with respect to the quiet Sun at $350 \mathrm{GHz}$, however no difference was found in the brightness distribution in the North-South from the East-West directions.

The purpose of this work is twofold: (i) study the temporal variation of the intensity and radial width of the polar

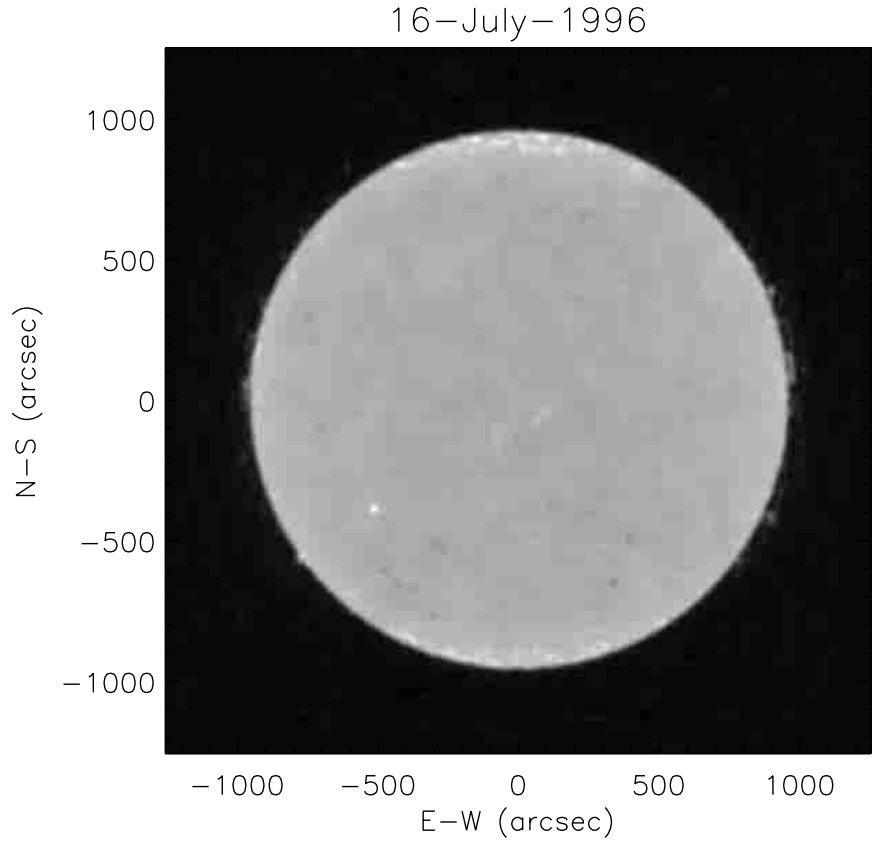

Fig. 1. Solar map from NoRH at 17 GHz observed on July 16, 1996.

temperature excess and their correlation with indicators of solar activity; and (ii) measure the brightness distribution as a function of position angle along the limb and over time in order to compare it with $\mathrm{H} \alpha$ and soft X-ray images, and faculae counts. The data are described in the next section, with the data analysis results presented in Sect. 3. Discussion of the results and main conclusions of this work are listed in Sect. 4.

\section{Observations}

A total of 3000 daily maps of the Sun, at $17 \mathrm{GHz}$ from the Nobeyama Radioheliograph (NoRH, Nakajima et al. 1994) from 1992 through 2001 were analyzed in this work. An example of such a map is shown in Fig. 1, obtained during a period of minimum activity. This period of observation is interesting because it starts just at the decline of one solar maximum and ends at the rise of the next maximum. At this radio frequency, it is believed that the emission originates at a height of approximately 3000 to $3500 \mathrm{~km}$ (Zirin 1988) corresponding to the high chromosphere or the base of the solar corona. The brightness temperature of the quiet Sun of every NoRH solar map is normalized to approximately $10^{4} \mathrm{~K}$ (Shibasaki 1998) according the atmosphere model proposed by Zirin et al. (1991).

In order to verify the existence of a correlation of the limb brightening with solar activity, the radio data will be compared with sunspot numbers (ftp . ngdc . noaa.gov/STP/ SOLAR_DATA/SUNSPOT_NUMBERS) and faculae counts. For the comparison with angular variation of the $17 \mathrm{GHz}$ limb, we use $10 \mathrm{H} \alpha$ images from the Big Bear Solar Observatory from August 1996, and soft X-ray images from Yohkoh from 1992-2000. 


\section{Data analysis and results}

From the synthesized NoRH images, we have extracted the brightness distribution profiles across the disc by making radial cuts through the center of the Sun, taken $1^{\circ}$ apart. The position angle of each profile is measured counter-clockwise with respect to the West equator. Each cut provided a brightness profile, where the increase near the limb is quite evident. Two quantities were chosen to characterize the excess brightening near the limb: its maximum intensity and its width. In order to measure the width, the intensity profile near the limb was fit by the following curve:

$f(x)=a x^{b} \exp \left(-c x^{d}\right)$

where $x$ is the angular position along the radial profile, and the width was defined at half maximum intensity. This procedure was performed in both studies of the temporal and angular variation of the limb brightening, as discussed in the following subsections.

\subsection{Temporal variation of the polar brightening}

Active regions are concentrated within a band on the solar disc, rarely occurring above $50^{\circ}$ latitude. Thus, in this study of the polar brightening, we will only consider the regions within $30^{\circ}$ of both North and South poles. This value was reached taking into account the $50^{\circ}$ maximum latitude of active regions plus the $7^{\circ}$ variation of the $B_{0}$ inclination angle (i.e., solar center latitude).

The polar intensity was estimated from the average of the 60 profiles taken between $60^{\circ}$ and $120^{\circ}$, and between $240^{\circ}$ and $300^{\circ}$ position angles $\left(90^{\circ}\right.$ and $270^{\circ}$ corresponding to the North and South poles, respectively). Figure 2a displays an example of such average profile, where the North and South polar brightening are clearly seen on the right and left of the profile, respectively. A zoom of the North region is shown in Fig. 2b with the fit given by Eq. (1) shown as a solid line. From this average profile, we obtain the maximum intensity and excess temperature width, taken at half the maximum value of the fit.

Only less than $1 \%$ of the maps were discarded because it was not possible to fit the data by Eq. (1). This occurs, in great part, due to the lack of brightening or due to the presence of a dark filament. From the remaining maps it was found that the constant $c$ in Eq. (1) is close to 1, resulting in an exponential increase of the temperature as the limb is approached.

Due to the variation of the $B_{0}$ angle, the values of intensity and width of the polar brightening were seen to oscillate with a period of 1 year. The Nobeyama data have been corrected for the position angle as an image rotation (as the $\mathrm{H} \alpha$ and Yohkoh images). However, it is not possible to correct the images for the $B_{0}$ angle oscillations without loss of data, whose yearly variation influences in some way the intensities measured. Thus, a Fourier filter was applied to the data eliminating the oscillations associated with periods of 1 year and 6 months.

The polar average intensities are shown in Fig. 3, where the a and c panels show the temporal variation of the Northern and Southern polar regions intensities, respectively. The mean maximum intensity for the North polar brightening was found
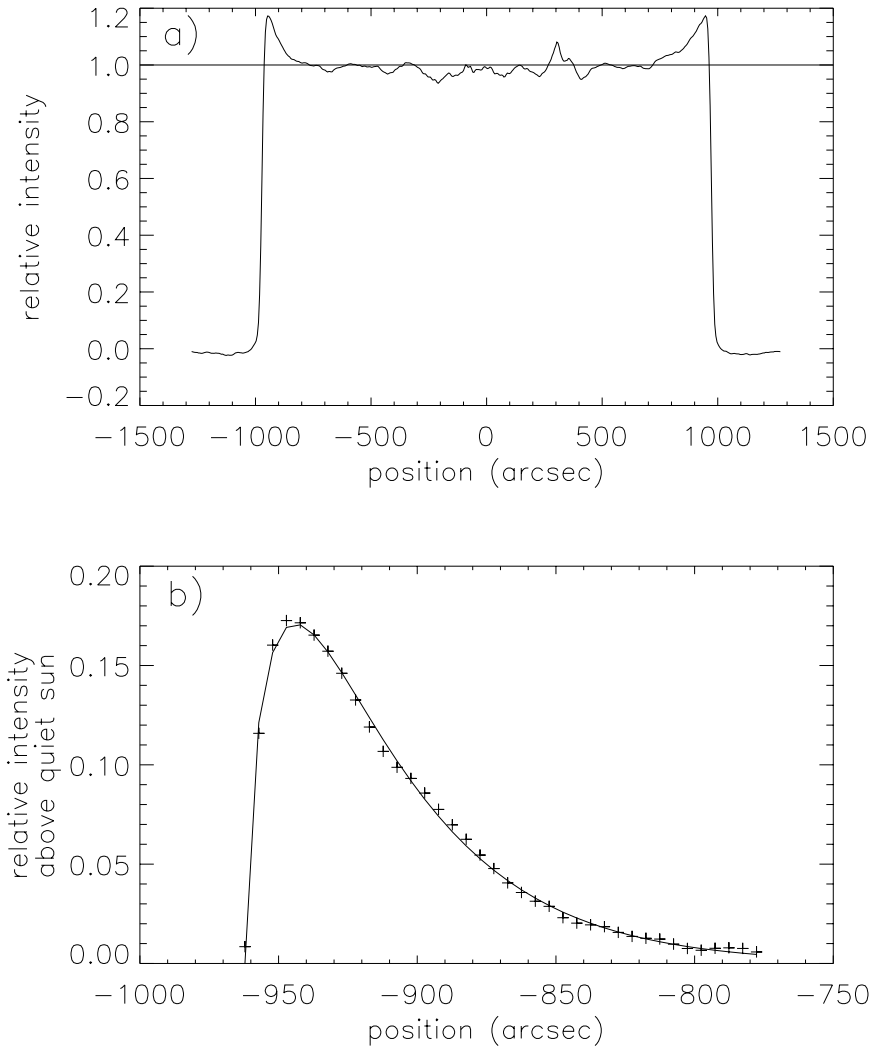

Fig. 2. a) Average of 60 scans within $\pm 30^{\circ}$ of the solar poles. b) Blow up of the Southern polar region shown in a), the solid line represents the fit by Eq. (1).

to be $13 \pm 4 \%$ above the quiet Sun value, whereas it was $14 \pm 4 \%$ for the South one, equivalent to a temperature excess of 1000$2000 \mathrm{~K}$. For comparison, the number of monthly-averaged daily polar faculae counts within latitudes of $70^{\circ}$ and $90^{\circ}$, as measured by Mitaka Observatory (Irie et al. 1993), are also shown in Figs. 3b, d for the North and South poles, respectively. A Fourier filter was also applied to the faculae data. A good coincidence in the overall temporal variation of the number of faculae and the polar radio intensity can be seen in the figure. Nevertheless, when compared with the number of sunspots plotted on panel e, an anti-correlation with solar activity is readily seen. For the sunspots, a correlation index of -0.81 was obtained for the Northern polar region and an index of -0.82 for the Southern one. Efanov et al. (1980) have also found an anti-correlation of the polar brightening with the solar cycle for short periods of data.

As for the width of the polar brightening, shown in Fig. 4, the mean values were $63 \pm 12 \operatorname{arcsec}$ and $69 \pm 16 \operatorname{arcsec}$ for the North and South polar regions, respectively. Only a small correlation with the solar cycle was found (correlation index of -0.14 and -0.17 for the North and South polar regions, respectively). What can be seen in the Figure is a greater variation of the width during the periods of maximum activity in 1992 and after 2000.

In order to verify if there were differences between both polar brightenings, we have plotted the intensities (Fig. 5a) and the widths (Fig. 5b) of the Southern region minus the Northern one. A running mean of 30 days is plotted as a thick line on 


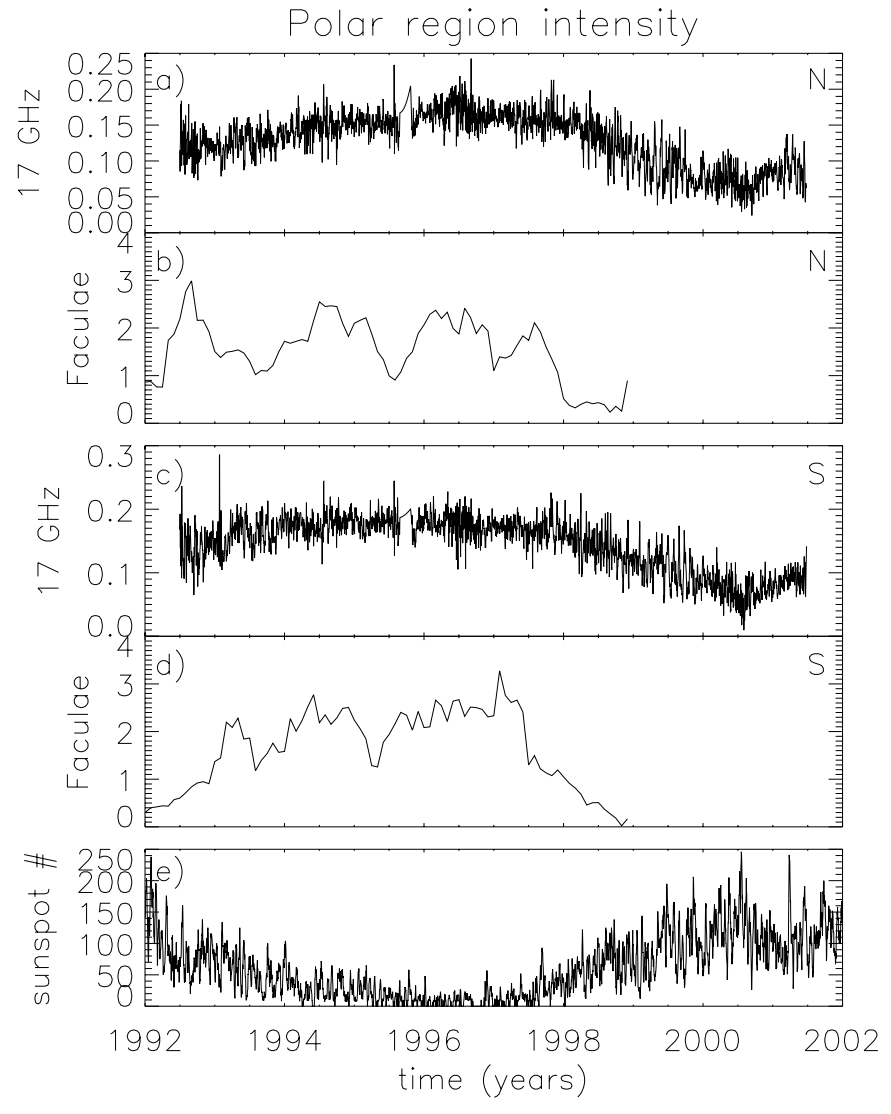

Fig. 3. Mean intensities of the brightness temperature above quiet Sun level at the poles for a) the Northern polar region and for c) the Southern polar region. Monthly average counts of polar faculae observed at the b) North and d) South poles. e) Sunspot number for the same period.

the figure. As can be seen from the plots in Fig. 5, the Southern polar region was both brighter and larger during the decline of last maximum from 1992 to 1996 . In order to check against the faculae data, Fig. 5c presents the South polar faculae number minus the North pole faculae. Even though the agreement is not as good as that of the radio intensities, there certainly is a predominance of faculae on the South pole over the North one during the period of 1993 through 1997, about the same period as the dominance of the Southern polar region intensity (panel a)). In summary, we found that during the decline of solar cycle 22, the Southern polar brightening was both more intense and occupied a larger area than the Northern one. It would be interesting to continue this study and see if this behavior reverses during the next activity cycle.

\subsection{Angular and temporal variation of the $\operatorname{limb}$ brightness}

In order to study the angular variation of the limb excess brightening, 10 maps made during the month of August of 1996, a period of minimum solar activity, were used. These maps were chosen such that there were also images available for the same days in soft X-rays from Yohkoh and $\mathrm{H} \alpha$ from the Big Bear Solar Observatory with no active regions close to the limb.
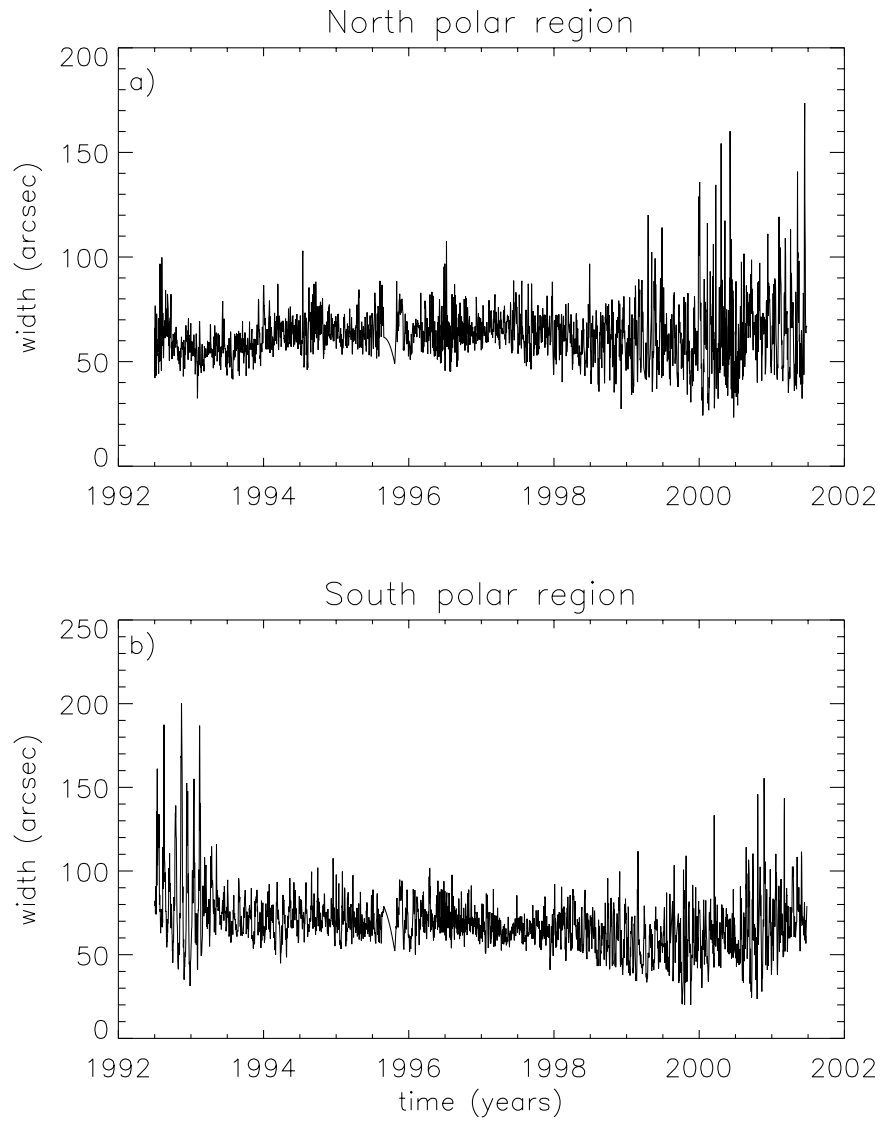

Fig. 4. Mean width of the polar excess temperature in arcsec for a) the Northern region and for $\mathbf{b})$ the Southern region.

From the $17 \mathrm{GHz}$ solar maps, radial cuts were taken every $2^{\circ}$. As in the case of the polar brightening study above, the limb brightness was taken as the maximum intensity close to the solar limb (with respect to quiet Sun value) for each radial profile corresponding to a position angle. The position angle is defined as before, i.e., the angle measured counter-clockwise from the West equator. The angular variation of limb intensity averaged for these 10 maps is displayed in Fig. 6a. As can be seen from this plot, the limb brightening is not uniformly distributed. Near the poles, the brightening is on average $25-27 \%$ above the quiet Sun value, whereas near the equatorial regions its mean value is $15 \%$. The intermediate regions have the smaller brightening, with average excess temperature of $10 \%$ above quiet Sun value. From a few days of observation during July 1984 at $36 \mathrm{GHz}$, Kosugi et al. (1986) found 3-7\% brightening at latitudes $>65^{\circ}$, whereas the brightening was less than $1 \%$ near the equator.

Photographic images have shown the presence of bright faculae near the poles of the Sun (Makarov \& Makarova 1996). Spicules are also observed in $\mathrm{H} \alpha$ images, being more numerous near the poles (Lippincott 1957; Rabin \& Moore 1980; Pataraya et al. 1990). As spicules and faculae are seen on $\mathrm{H} \alpha$ images, we have made a detailed comparison between these images and the $17 \mathrm{GHz}$ maps. Since previous work also suggested that the polar brightenings were associated with coronal holes, we have analyzed Yohkoh soft X-ray images as well. 

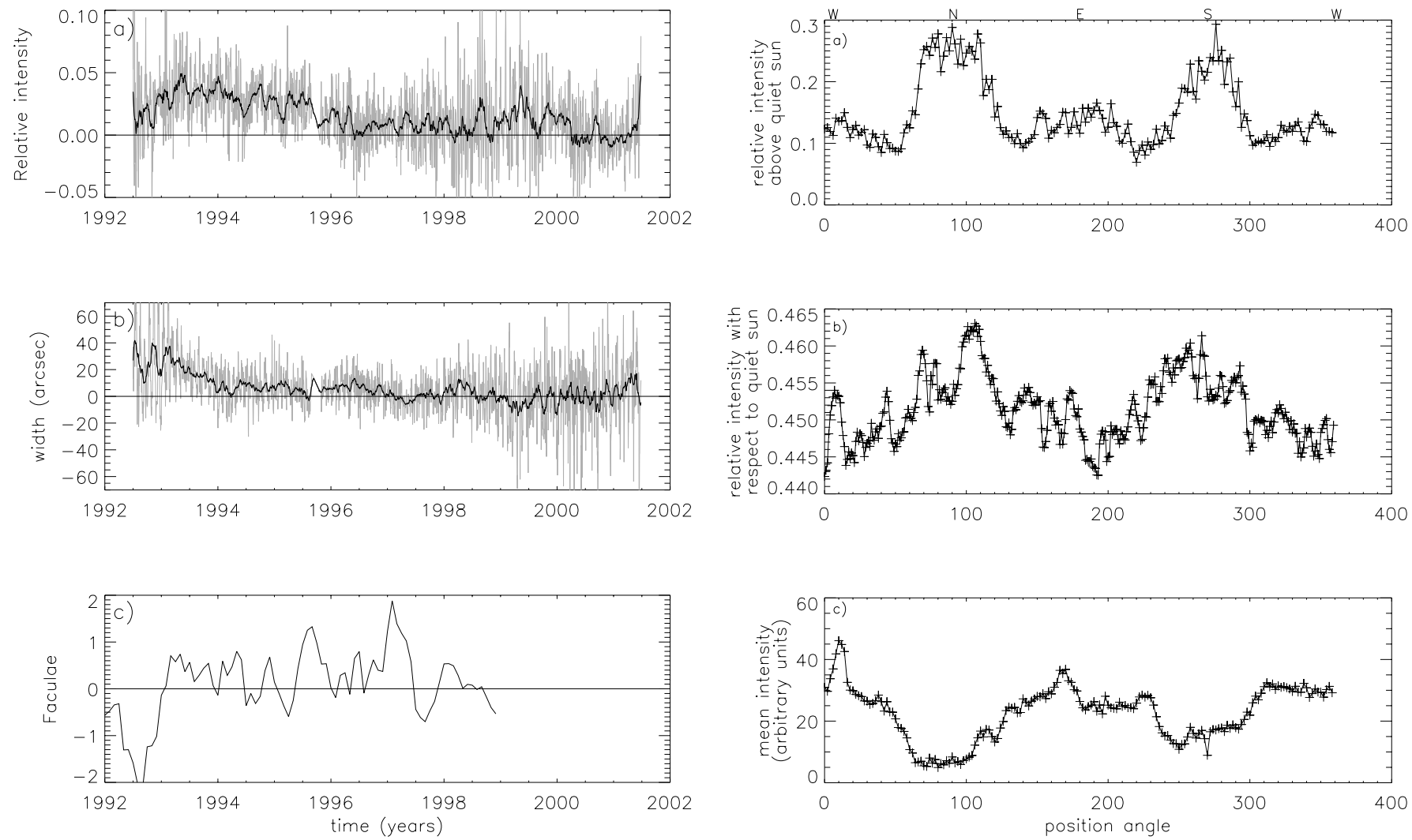

Fig. 5. The difference in a) intensity (above quiet Sun levels) and b) width (in arcsec) for the brightening of Southern polar region minus the Northern one. c) The difference in the number of faculae detected on the South pole minus those on the North pole for the same period.

The analysis of $\mathrm{H} \alpha$ images consisted of three steps: (i) determining the quiet Sun intensity as the most probable value of the image/map, (ii) extracting radial profiles of the image every $1^{\circ}$, and (iii) defining the mean intensity at that angle by averaging the intensity at the limb, from 0.99 to 1.01 of the solar radius. The limb was taken at half the quiet Sun value. An example of such analysis is shown in Fig. 6b, for $10 \mathrm{H} \alpha$ images obtained for the same days of August 1996 as the radio maps. An increase in intensity of about $1-2 \%$ at both polar regions is clearly seen in the figure.

Usually, the polar regions seen in soft X-ray images are dark regions of much smaller emission wherever coronal holes are found. Next we studied a possible association between the coronal holes and the bright polar regions seen in microwaves. In order to do so, the solar limb of soft X-ray images were adjusted for circumference, and the mean intensity was taken from $25^{\prime \prime}$ inside to $25^{\prime \prime}$ just outside the limb. An example of such a result is presented in Fig. 6c for the same days of August 1996. During this period, a decline in intensity at the North pole due to the presence of a coronal hole is quite evident.

A comparison of the limb angular profile at the three wavelengths shows that the presence of coronal holes at the poles, seen in the X-ray images, is associated with an increase in intensity in the $17 \mathrm{GHz}$ and $\mathrm{H} \alpha$ emission. In the X-ray images for this period, the North coronal hole is more prominent than the Southern one. Correspondingly, the data at the other two

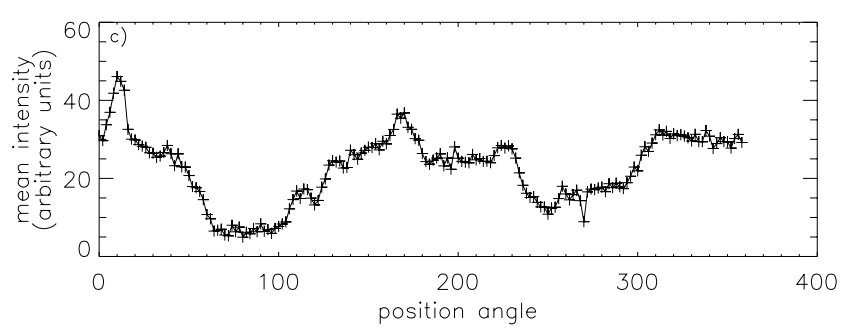

Fig. 6. Angular variation of the limb brightening as a function of position angle for a) $17 \mathrm{GHz}$ maps, b) $\mathrm{H} \alpha$, and c) soft X-ray images. These are mean values of images taken during 10 days of August 1996, at the three wavelengths.

wavelengths show that the emission at the North pole is greater than that at the Southern one. An enhancement of the emission at the equatorial regions is seen at all three wavelengths.

In order to study the temporal evolution of the limb brightening during the solar cycle, we have performed the same analysis described above for a series of $17 \mathrm{GHz}$ and soft X-ray maps, from July 1992 through December 2000. Unfortunately, there is no such continuous coverage of high resolution $\mathrm{H} \alpha$ images for the same period.

Once a month during this period, a $17 \mathrm{GHz}$ and a soft X-ray image, for the same day, were sliced every $2^{\circ}$. The limb brightening as a function of position angle was calculated as described previously. The results for all the 88 maps analyzed are plotted in Figs. 7 and 8, for $17 \mathrm{GHz}$ and soft X-ray, respectively. These figures show the intensity, as gray scale, plotted as position angle ( $y$ axis) versus time ( $x$ axis). Hence, each column of the images represents the brightening as a function of position angle for a single day. Note that the $17 \mathrm{GHz}$ brightening is that above the quiet Sun value.

Two features are clearly distinguished in each figure. The two continuous bright bands seen in Fig. 7 outline the brightening at the North and South poles. Moreover this brightening increases during periods of minimum solar activity. The bright regions corresponding to active regions near the East and West equatorial limbs are seen to follow the known butterfly diagram, approaching the equator as the cycle evolves. Also seen 


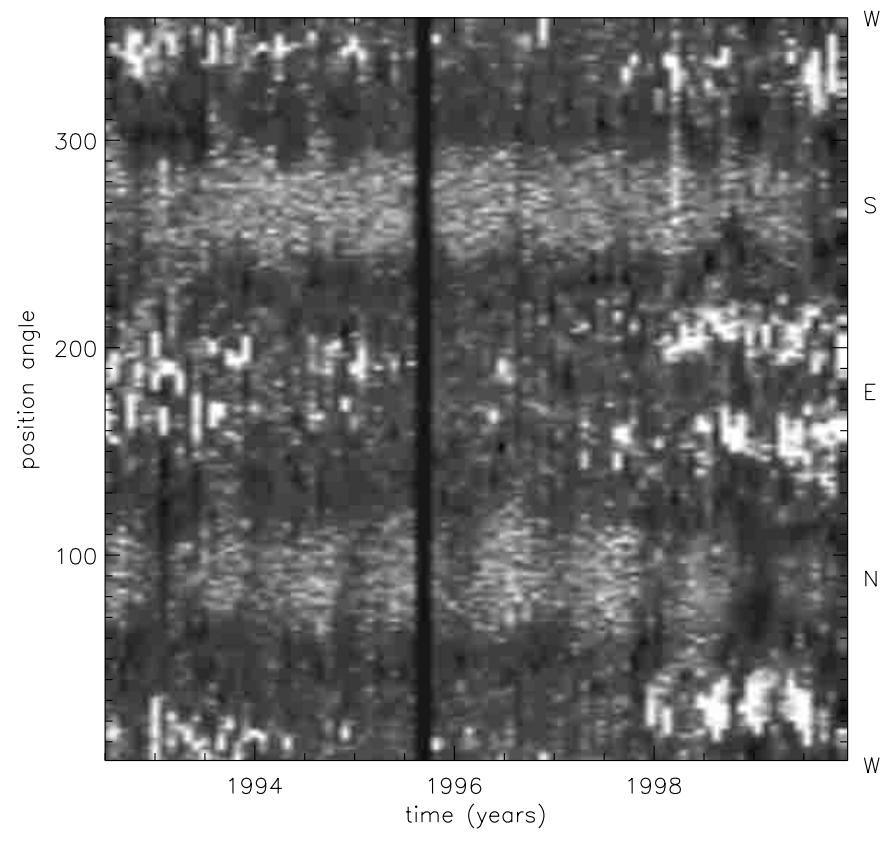

Fig. 7. Temporal evolution of the $17 \mathrm{GHz}$ angular variation of the limb brightening. North pole corresponds to $90^{\circ}$ position angle, whereas South corresponds to $270^{\circ}$.

in Fig. 7 is the yearly variation of polar brightening due to the change in $B_{0}$.

The temporal evolution of the solar limb seen in soft $\mathrm{X}$-ray images is similar to the radio limb, as far as the equatorial regions are concerned, where there is a strong correlation between the active regions seen at soft X-ray and $17 \mathrm{GHz}$. However, the polar regions are quite distinct. These polar regions (position angles around $90^{\circ}$ and $270^{\circ}$ ) are seen as dark bands across the figure, indicating the presence of coronal holes. Even though a one-to-one correspondence between coronal hole and microwave brightening cannot always be found, the microwave brightenings statistically correlate with the coronal holes in the polar regions over the period of approximately one solar cycle.

\section{Discussion and conclusions}

Our results have shown that the emission close to the solar limb is far from being uniform at $17 \mathrm{GHz}$, either spatially or in time. Our main results may be summarized as:

1. The mean intensity of the polar regions was found to be approximately $13 \%$ and $14 \%$ above quiet Sun levels at the North and South poles, respectively.

2. The polar brightenings are anti-correlated with the solar cycle, being strongest during periods of minimum activity.

3. The width of the excess temperature was observed to be slightly over 1 arcmin for both polar regions.

4. The Southern polar brightening was found to be both wider and brighter during the decline of the previous maximum.

5. There is an excess temperature all around the limb and not just at the poles. During periods of minimum activity, this

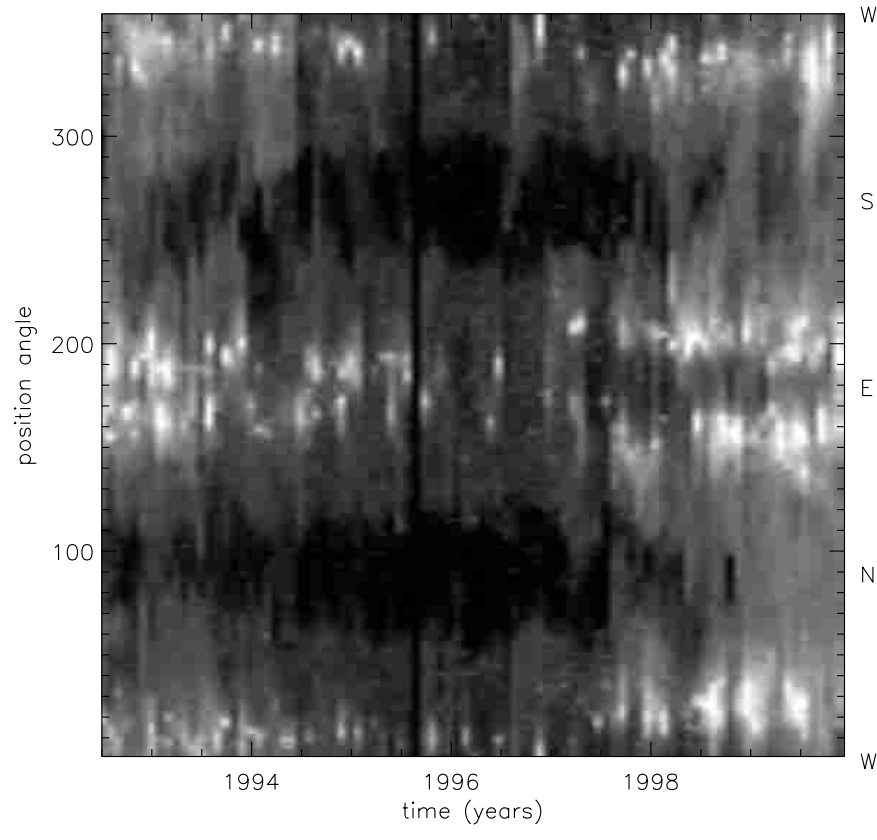

Fig. 8. Same as the previous figure for the soft X-ray images from Yohkoh.

brightening above quiet Sun level reaches $25 \%$ at the polar regions, $\sim 15 \%$ near the equator, and $10 \%$ at intermediate regions.

6. During periods of minimum activity, a strong correlation exists between the angular variation of the limb brightening observed in the radio and $\mathrm{H} \alpha$ images. The poles are also the regions with strongest emission at $\mathrm{H} \alpha$.

7. There is also a strong correlation of the radio maps with soft $\mathrm{X}$-ray images at the equatorial regions due to the presence of active regions, especially during periods of solar maximum activity. However, the radio polar brightenings were strongly anti-correlated with the coronal holes seen in soft $\mathrm{X}$-ray images.

An increase of the emission is seen at the polar regions, as had been previously pointed out by Shibasaki (1998), Kosugi et al. (1986), and Nindos et al. (1999) from analysis of North-South scans. Moreover the polar brightenings are anti-correlated with the solar cycle, being strongest during periods of minimum activity. This result agrees with that of Efanov et al. (1980) obtained at millimetric wavelengths, thus suggesting that the polar brightening is dependent on the phase of the solar cycle, being anti-correlated with sunspot number. A good correlation with the number of polar faculae was also found (Fig. 3).

The mean intensity of the polar regions reported here ( 1300-1400 K, for a quiet Sun brightness temperature of $10^{4} \mathrm{~K}$ ) is consistent with the results of Nindos et al. (1999). The authors found the polar brightening to consist of a diffuse component of about $1500 \mathrm{~K}$ excess temperature with patchy sources of more than $3000 \mathrm{~K}$ above quiet Sun levels, basically the same result found as by Shibasaki (1998) and Koshiishi (1996).

We would like to point out that this work is the first to analyze the limb brightening as a function of position angle, and 
also in time. Here we have shown that there is an excess temperature all around the limb and not just at the poles. The temperature enhancement is not uniform all around the limb, being strongest at the poles and weakest at intermediate latitudes.

As for the comparison with observations at other wavelengths, the good correspondence of the radio brightness enhancements with $\mathrm{H} \alpha$ seem to indicate that the emission at $17 \mathrm{GHz}$ is formed at chromospheric heights. As for the association of polar-cap brightening at radio frequencies with coronal holes that had been pointed out before by Shibasaki (1998) at $17 \mathrm{GHz}$ and by Kosugi et al. (1986) suggests that they are due to the temperature and density structure of the upper chromosphere in these regions (Kosugi et al. 1986). However, Shibasaki (1998) did not verify a one-to-one correlation between the polar brightening at $17 \mathrm{GHz}$ and coronal holes observed in soft X-ray images.

Results 1, 2, 4, and 5 listed above suggest an association between the limb brightening observed at $17 \mathrm{GHz}$ and the presence of faculae. The main observational evidence is as follows. First, faculae are known to be more abundant near the poles (Erofeev 1980; Unruh et al. 1999; Ortiz et al. 2002), however they are also present in active regions (Ortiz et al. 2002). The study of radio limb brightening as a function of position angle has shown that the emission is stronger at the polar region and also at the equatorial limb (Fig. 6), albeit less intense, thus agreeing with the faculae distribution. Second, at polar regions the number of faculae is seen to be anti-correlated with the sunspot cycle (Makarov \& Makarova 1996, had already pointed out that the number of polar faculae is observed to be anti-correlated with the sunspot cycle), the same behavior was detected for the $17 \mathrm{GHz}$ polar brightenings (Fig. 3). Moreover, there seems to have a predominance of the faculae number of one pole over the other for different cycles. During the decline of cycle 22, both the number of faculae and the radio polar emission was larger for the South pole (Fig. 5). Results on surface densities of polar faculae from Erofeev et al. (1980) also seem to show a dominance of one pole over the other for each cycle (see their Fig. 3).

A possible cause of the association between the excess brightness at $17 \mathrm{GHz}$ and faculae may be explained as follows. Only a fraction of the limb brightening predicted by theoretical models is observed at radio frequencies. Several authors (Simon \& Zirin 1969; Wannier et al. 1983) have claimed the spicules as responsible for the decrease in emission at radio wavelengths. This occurs because of their lower temperature and higher density than the surrounding atmosphere where the emission at these frequencies originates. It is known that there are no spicules over plages (Zirin 1988), due to the sharp transition in temperature from the chromosphere to the corona. Since faculae are small plage regions, or plagettes, probably no spicules occur above them either. Therefore, the regions above faculae would appear brighter at $17 \mathrm{GHz}$ because of the absence of spicules, thereby showing higher chromospheric emission due to its positive temperature gradient. The surrounding regions, on the other hand, would have their emission partly obscured by the spicules. This may be the interpretation of the bright patchy sources identified by Shibasaki (1998).
Thus, the angular variation of the $17 \mathrm{GHz}$ limb intensity, as shown in Fig. 6, may be explained as follows. All around the solar limb, there is an effective brightness excess of $10 \%$, very likely due to the positive temperature gradient, already taking into account the presence of spicules. This is what is observed at the intermediate regions between the poles and the equatorial limb. The extra 15-20\% brightness temperature enhancement above the $10 \%$ level and the quiet Sun level are associated with the polar faculae seen at optical wavelength near the poles. Their good temporal correlation was already pointed out above. At the equatorial limb regions (within latitudes $\pm 30^{\circ}$ ), since these are the sunspot belt zones, the radio enhancements of $5 \%$ (on top of the isotropic $10 \%$ brightening) are associated with features of the same type as active region faculae, even if there were no active regions right at the limb during the 10 days of solar minimum images analyzed. The influence of spicules and faculae on the observed radio brightness temperature will be further investigated in a future work.

Acknowledgements. We are grateful to the BBSO observing staff for obtaining the full disk $\mathrm{H} \alpha$ images. CLS acknowledges support from the Brazilian agency FAPESP, grant number 01/02106-3. Yohkoh is operated by ISAS, NASA and Yohkoh team members. The Nobeyama Radioheliograph is operated by NAOJ/Nobeyama Solar Radio Observatory.

\section{References}

Bastian, T. S., Ewell, M. W., \& Zirin, H. 1993, ApJ, 415, 364

Belkora, L., Hurford, G. J., Gary, D. E., \& Woody, D. P. 1992, ApJ, 400, 692

Chapman, G. 1979, ApJ, 232, 923

Clark, C. D., \& Park, W. M. 1968, Nature, 219, 922

Efanov, V. A., Moissev, I. G., Nesterov, N. S., \& Stewart, R. T. 1980, in Radio Physics of the Sun, ed. M. R. Kundu, \& T. E. Gergely (Dordrecht: Reidel), IAU Symp., 86, 141

Erofeev, D. V. 2001, Sol. Phys., 203, 9

Ewell, M. W. Jr., Zirin, H., Jensen, J. B., \& Bastian, T. S. 1993, ApJ, 403, 426

Furst, E., Hirth, W., \& Lantos, P. 1979, Sol. Phys., 63, 257

Gelfreikh, G. B., Makarov, V. I., Tlatov, A. G., Riehokainen, A., \& Shibasaki, K. 2002, A\&A, 389, 618

Gomez-Gonzalez, J., Barcia, A., Delgado, A., \& Planesas, P. 1983, A\&A, 122, 219

Horne, K., Hurford, G. J., Zirin, H., \& de Graauw, Th. 1981, ApJ, 244, 340

Irie, et al. 1993, Rep. NAOJ, 2, 403, http://solarwww.mtk.nao.ac.jp

Joensen, P., McCutcheon, W. H., \& Shuter, W. L. H. 1974, Sol. Phys., 39, 309

Kawabata, K., Fujishita, M., Kato, T., Ogawa, H., \& Omodaka, T. 1980, Sol. Phys., 65, 221

Koshiishi, H. 1996, Ph.D. Thesis, University of Tokyo, Japan

Kosugi, T., Ishiguro, M., \& Shibasaki, K. 1986, PASJ, 38, 1

Kundu, M. R., \& McCullough, T. P. 1972, Sol. Phys., 24, 133

Kundu, M. R., Sou-Yang, L., \& McCullough, T. P. 1977, Sol. Phys., 51, 321

Lindsey, C., de Graauw, T., de Vries, C., \& Lidholm, S. 1984, ApJ, 277, 424

Lindsey, C., Hildebrand, R. H., Keene, J. K., \& Whitcomb, S. E. 1981, ApJ, 248, 830 
Lindsey, C., Kopp, G., Clark, T. A., \& Watt, G. 1995, ApJ, 453, 511

Lippincott, S. L. 1957, Smithsonian Contr. Ap., 2, 15

Makarov, V. I., \& Makarova, V. V. 1996, Sol. Phys., 163, 267

Nakajima, H., Nishio, M., Enome, S., et al. 1994, Proc. IEEE, 82, 705

Nindos, A., Kundu, M. R., White, M., et al. 1999, ApJ, 527, 415

Ortiz, A., Solanki, S. K., Doming, V., Fligge, \& Sanahuja, B. 2002, A\&A, 388, 1036

Pataraya, A. D., Taktakishvili, A. L., \& Chargeishvili, B. B. 1990, Sol. Phys., 128, 333

Pohjolainen, S. 2000, A\&A, 361, 349

Rabin, D., \& Moore, R. L. 1980, ApJ, 241, 394

Riehokainen, A., Urpo, S., \& Valtaoja, E. 1998, A\&A, 333, 741

Riehokainen, A., Urpo, S., Valtaoja, E., et al. 2001, A\&A, 366, 676
Shibasaki, K. 1998, in Synoptic Solar Physics, ed. K. S. Balasubramaniam, J. Harvey, \& D. Rabin, ASP Conf. Ser., 140, 373

Shimabukuro, F. I., Wilson, W. J., Mori, T. T., \& Smith, P. L. 1975, Sol. Phys., 40, 359

Simon, M., \& Zirin, H. 1969, Sol. Phys., 9, 317

Spruit, H. C. 1976, Sol. Phys., 50, 269

Unruh, Y. C., Solanki, S. K., \& Fligge, M. 1999, A\&A, 345, 635

Wannier, P. G., Hurford, G. J., \& Seielstad, G. A. 1983, ApJ, 264, 660

Zirin, H., Baumert, B. M., \& Huford, G. J. 1991, ApJ, 370, 779

Zirin, H. 1988, in Astrophysics of the Sun (New York, EUA: Cambridge University Press) 